\title{
INVARIANT-FREE REPRESENTATIONS OF AUGMENTED RINGS
}

BY

\author{
PETER M. CURRAN
}

\begin{abstract}
Let $\Gamma$ be an augmented ring in the sense of Cartan-Eilenberg, and let there be given a representation of $\Gamma$ in $\operatorname{End}_{k} A$, where $A$ is a finite dimensional vector space over the field $k$. We show that all cohomology of $\Gamma$ in $A$ is trivial if there are no invariants in $A$ under the action of a suitable commutative subring of $\Gamma$. This generalizes a previous result of the author for group cohomology, and is applied to obtain sufficient conditions for the vanishing of the cohomology of Lie algebras and associative algebras.
\end{abstract}

Introduction. Throughout this paper, $\Gamma$ will be a (left) augmented ring in the sense of Cartan-Eilenberg [2, Chapter VIII]:

$$
0 \rightarrow I_{\Gamma} \rightarrow \Gamma \stackrel{\varepsilon_{\Gamma}}{\rightarrow} Q_{\Gamma} \rightarrow 0,
$$

and all subrings will contain the unity element of $\Gamma$. (The subscripts $\Gamma$ will usually be omitted.) Clearly, any subring is also an augmented ring. If $A$ is a (left) $\Gamma$-module, then

$$
A^{\Gamma}=\{a \in A: I a=0\}
$$

is called the set of invariant elements of the representation.

The main purpose of this paper is to show that if $A$ is a finite dimensional vector space, or more generally, a module which can be obtained inductively by repeated extensions of such spaces, and $A^{\Lambda}=0$ for a suitable commutative subring $\Lambda$ of $\Gamma$, then all cohomology of $\Gamma$ in $A$ is trivial (Theorem 1). We thus obtain results about the cohomology, and hence about extensions, of groups, Lie algebras and associative algebras. In particular, the results of [3] are subsumed here. The application to Lie algebras (Corollary 1) should be compared with a theorem of D. W. Barnes [1] (and an earlier special case due to Dixmier [4]) to the effect that all cohomology of a finite dimensional nilpotent Lie algebra $L$ in an $L$-module $A$ vanishes if $A^{L}=0$.

Received by the editors October 31, 1975.

AMS (MOS) subject classifications (1970). Primary 18H15, 18H25, 16A62, 16A64, 17B10, 17B55; Secondary 16A56, 18H10, 20C05, $20 \mathrm{~J} 05$.

Key words and phrases. Augmented ring, cohomology of Lie algebras, cohomology of associative algebras, extensions of Lie algebras, extensions of associative algebras.

- American Mathematical Society 1977 
The following sample illustrates the application of the main theorem to extension theory.

THEOREM. Let $E$ be a group (resp., Lie algebra over a field, resp., associative algebra over $a$ field) and let $A$ be a normal abelian subgroup which is a direct sum of a finite group and a finite dimensional rational vector space (resp., finite dimensional abelian ideal, resp., finite dimensional square zero ideal) in $E$ such that $E / A$ is abelian. If the center of $E$ is disjoint from $A$, then $E$ splits over $A$ (Corollaries 2 and 5, and [3, Corollary 3]).

1. The central case. The proof of the following lemma is similar to that of statement (b), $\$ 1$ of [3].

LEMMA 1. Let $\Gamma$ be an augmented ring with center $Z$, and let $A$ be a left $\Gamma$ module. If there is an element $\alpha \in Z \cap I$ such that the endomorphism of $A$ given by $a \mapsto \alpha a$ is bijective, then

$$
\operatorname{Ext}_{\Gamma}^{r}(Q, A)=0 \text { for } r \geqslant 0 .
$$

Proof. Start with a $\Gamma$-projective resolution of $Q$ of the form

$$
\cdots \rightarrow X_{1} \rightarrow \Gamma \stackrel{\&}{\rightarrow} Q \rightarrow 0,
$$

and observe that the endomorphism of this complex determined by $x \mapsto \alpha x$ is a map over the zero map on $Q$. Then proceed as in [3].

Proposition 1. Let $\Gamma$ be an augmented ring with center $\Lambda, k$ a field, and $A$ a $\Gamma$ - $k$-bimodule, finite dimensional over $k$. Suppose that at least one of the following conditions is satisfied:

(a) $\Gamma$ is a k-algebra (in such a way that $(c \gamma) a=c(\gamma a)$ for all $c \in k, \gamma \in \Gamma, a$ $\in A)$.

(b) $k_{0}$, the prime subfield of $k$, has order $>\operatorname{dim}_{k} A$.

(c) Every $I_{\Lambda}$-invariant $k$-subspace of $A$ is $\Gamma$-invariant.

Then, if $A^{\Lambda}=0$,

$$
\operatorname{Ext}_{\Gamma}^{r}(Q, A)=0 \text { for } r \geqslant 0 .
$$

Proof. (a) We assume first that $\operatorname{dim}_{k} A=n<q=|k|$, the order of $k$. For $\lambda \in \Lambda$, let $e_{\lambda}$ be the $k$-endomorphism of $A$ given by: $e_{\lambda} a=\lambda a$. Let

$$
B=\left\{e_{\lambda}+1: \lambda \in I_{\Lambda}\right\} \subset \operatorname{End}_{k} A .
$$

By part (b) of the Lemma, $\$ 2$ of [3], there exist $\lambda_{i} \in I_{\Lambda}$ and $c_{i} \in k$ such that $\sum c_{i} e_{\lambda_{i}}$ is invertible. Hence $\alpha=\sum c_{i} \lambda_{i}$ satisfies the hypothesis of Lemma 1, so the desired conclusion follows. 
If $n \geqslant q$, let $L$ be an extension of $k$ with $|L|>n$. Then tensor $\Gamma, Q$, and $A$ with $L$ (over $k$ ) and apply the preceding discussion to conclude that

$$
\operatorname{Ext}_{L \otimes_{k} \Gamma}^{r}\left(L \otimes_{k} Q, L \otimes_{k} A\right)=0 \text { for } r \geqslant 0 .
$$

(One must check that $\left(L \otimes_{k} A\right)^{\Lambda^{\prime}}=0$, where

$$
\Lambda^{\prime}=\operatorname{Im}\left(L \otimes_{k} \Lambda \rightarrow L \otimes_{k} \Gamma\right)
$$

Suppose

$$
\beta=\sum l_{i} \otimes a_{i} \in\left(L \otimes_{k} A\right)^{\Lambda^{\prime}},
$$

where we may assume the $l_{i}$ linearly independent over $k$. Then for any $\lambda \in I_{\Lambda}$,

$$
0=(1 \otimes \lambda) \beta=\sum l_{i} \otimes \lambda a_{i} .
$$

Hence each $\lambda a_{i}=0$, so $a_{i}=0$.)

Now by [2, VIII, Theorem 3.1],

$$
\operatorname{Ext}_{L \otimes_{k} \Gamma}^{r}\left(L \otimes_{k} Q, L \otimes_{k} A\right) \approx \operatorname{Ext}_{\Gamma}^{r}\left(Q, L \otimes_{k} A\right) .
$$

(Condition (i) of that theorem follows from [2, II, Proposition 5.1], and (ii) follows from [2, II, Proposition 6.1] with $\phi: k \rightarrow \Gamma$ the obvious map.)

Finally, let $\sigma: L \rightarrow k$ be a $k$-linear map such that the composite $k \rightarrow L \rightarrow k$ is the identity. Then the composite

$$
A \stackrel{i}{\rightarrow} L \otimes_{k} A \stackrel{j}{\rightarrow} A,
$$

where $i a=1 \otimes a$ and $j(l \otimes a)=\sigma(l) a$, is also the identity, and $i$ and $j$ are $\Gamma$ maps, so the composite

$$
\operatorname{Ext}_{\Gamma}^{r}(Q, A) \rightarrow \operatorname{Ext}_{\Gamma}^{r}\left(Q, L \otimes_{k} A\right) \rightarrow \operatorname{Ext}_{\Gamma}^{r}(Q, A)
$$

is the identity. Now (1) and (2) imply that $\operatorname{Ext}_{\Gamma}^{r}(Q, A)=0$.

(b) We proceed as in (a) to find a $k$-automorphism of $A$ of the form $\sum c_{i} e_{\lambda_{i}}$ with $c_{i} \in k$ and $\lambda_{i} \in I_{\Lambda}$, and it is clear from the proof of the Lemma, $\$ 2$ of [3], that the $c_{i}$ can be chosen as "integers": $c_{i}=n_{i} \cdot 1 \in k_{0}\left(n_{i} \in \mathrm{Z}\right)$ since $\left|k_{0}\right|>\operatorname{dim}_{k} A$. Then $\alpha=\sum n_{i} \lambda_{i}$ satisfies the hypothesis of Lemma 1 .

(c) Let $A=\sum A_{i}$ be a direct sum decomposition of $A$ into indecomposable $I_{\Lambda}$-invariant $k$-subspaces. By hypothesis (c), each $A_{i}$ is a $\Gamma$-submodule, so it suffices to show that for all $r \geqslant 0$,

$$
\operatorname{Ext}_{\Gamma}^{r}\left(Q, A_{i}\right)=0 \text { for each } i .
$$

By part (a) of the Lemma, $\$ 2$ of [3], with $V=A_{i}$ and 


$$
B=\left\{e_{\lambda}+1: \lambda \in I_{\Lambda}\right\} \subset \operatorname{End}_{k} A_{i}
$$

$\left(e_{\lambda} a=\lambda a\right)$, there exists $\alpha \in I_{\Lambda}$ such that $e_{\alpha}$ is invertible. Now an application of Lemma 1 completes the proof.

2. The general case. Let $\Lambda$ and $\Gamma$ be augmented rings and let $\phi: \Lambda \rightarrow \Gamma$ be a ring homomorphism such that $\phi\left(I_{\Lambda}\right) \subset I_{\Gamma}$. We proceed as in [2, XVI, §6] to obtain a spectral sequence, (4) below, which will be needed to extend Proposition 1.

We call $\phi$ (right) normal if the left ideal $\Gamma \phi\left(I_{\Lambda}\right)$ of $\Gamma$, denoted $\Gamma \cdot I_{\Lambda}$, is also a right ideal. Then

$$
\Gamma^{\prime}=\Gamma / \Gamma \cdot I_{\Lambda} \stackrel{e^{\prime}}{\rightarrow} Q_{\Gamma} \rightarrow 0
$$

is an augmented ring if we define $\varepsilon^{\prime}$ by

$$
\varepsilon^{\prime}\left(\gamma+\Gamma \cdot I_{\Lambda}\right)=\varepsilon_{\Gamma}(\gamma)
$$

( $Q_{\Gamma}$ is a left $\Gamma^{\prime}$-module since $\Gamma \cdot I_{\Lambda}$ annihilates $Q_{\Gamma}$, viz., for $\alpha \in \Gamma \cdot I_{\Lambda}$ and $q=\varepsilon_{\Gamma} \gamma_{1} \in Q_{\Gamma}$, we have $\alpha q=\varepsilon_{\Gamma}\left(\alpha \gamma_{1}\right)$, which is 0 since $\Gamma \cdot I_{\Lambda}$ is a right ideal.)

Now the sequence

$$
0 \rightarrow I_{\Lambda} \rightarrow \Lambda \rightarrow Q_{\Lambda} \rightarrow 0
$$

yields

$$
\Gamma \otimes_{\Lambda} I_{\Lambda} \rightarrow \Gamma \rightarrow \Gamma \otimes_{\Lambda} Q_{\Lambda} \rightarrow 0
$$

so

$$
\Gamma \otimes_{\Lambda} Q_{\Lambda} \approx \Gamma^{\prime}
$$

Then the spectral sequence $(2)_{4}$, Case 4 , of $[2, X V I, \S 5]$ becomes in our case:

$$
\operatorname{Ext}_{\Gamma^{\prime}}^{p}\left(Q_{\Gamma}=Q_{\Gamma^{\prime}}, \operatorname{Ext}_{\Gamma}^{q}\left(\Gamma^{\prime}, A\right)\right) \Rightarrow \operatorname{Ext}_{\Gamma}^{n}\left(Q_{\Gamma}, A\right)
$$

for any left $\Gamma$-module $A$.

Now suppose $\Gamma$ is projective as a right $\Lambda$-module (via $\phi)$. Then by [2, VI, Proposition 4.1.3] with $A$ and $C$ replaced by $Q_{\Lambda}$ and $A$, resp.,

$$
\operatorname{Ext}_{\Gamma}^{q}\left(\Gamma^{\prime}, A\right) \approx \operatorname{Ext}_{\Gamma}^{q}\left(\Gamma \otimes_{\Lambda} Q_{\Lambda}, A\right) \approx \operatorname{Ext}_{\Lambda}^{q}\left(Q_{\Lambda}, A\right),
$$

so (3) becomes

$$
\operatorname{Ext}_{\Gamma^{\prime}}^{p}\left(Q_{\Gamma}=Q_{\Gamma^{\prime}}, \operatorname{Ext}_{\Lambda}^{q}\left(Q_{\Lambda}, A\right)\right) \Rightarrow \operatorname{Ext}_{\Gamma}^{n}\left(Q_{\Gamma}, A\right) .
$$

In what follows, a subring $\Lambda$ of an augmented ring $\Gamma$ will be called normal if the inclusion $\Lambda \rightarrow \Gamma$ is normal and $\Gamma$ is projective as a right $\Lambda$-module. $\Lambda$ is subnormal if there is a finite sequence 


$$
\Lambda=\Lambda_{0} \subset \Lambda_{1} \subset \cdots \subset \Lambda_{t}=\Gamma
$$

with $\Lambda_{i}$ normal in $\Lambda_{i+1}$ for each $i$. For example, if $N$ is a normal subgroup of the group $G$, then $\mathbf{Z} N$ is a normal subring of $\mathbf{Z} G$ (see [2, XVI, §6]). Similarly, if $J$ is an ideal in the Lie algebra $L$ (over a field), then $J^{e}$, the enveloping algebra of $J$, is normal in $L^{e}$ [ibid.].

THEOREM 1. Let $\Gamma$ be an augmented ring, $k$ a field, and $A$ a $\Gamma$ - $k$-bimodule, finite dimensional over $k$. Let $\Lambda$ be a subnormal commutative subring of $\Gamma$, and suppose at least one of the following conditions is satisfied (see Proposition 1 for notation):

(a) $\Lambda$ is a $k$-algebra $($ with $(c \lambda) a=c(\lambda a))$,

(b) $\left|k_{0}\right|>\operatorname{dim}_{k} A$,

(c) Every $I_{\Lambda}$-invariant $k$-subspace of $A$ is $\Lambda$-invariant. If $A^{\Lambda}=0$, then

$$
\operatorname{Ext}_{\Gamma}^{r}(Q, A)=0 \text { for } r \geqslant 0 .
$$

Proof. Given the sequence (5), let $Q_{i}=Q_{\Lambda_{i}}=\varepsilon_{\Gamma}\left(\Lambda_{i}\right)$. By Proposition 1, $\operatorname{Ext}_{\Lambda_{i}}^{r}\left(Q_{i}, A\right)=0$ for all $r$ when $i=0$. Now apply the spectral sequence (4) (with $\Lambda_{i}$ and $\Lambda_{i+1}$ in place of $\Lambda$ and $\Gamma$ ) to conclude inductively that for all $r$,

$$
\operatorname{Ext}_{\Lambda_{i}}^{r}\left(Q_{i}, A\right)=0 \text { for } i=0,1, \ldots, t .
$$

Remark. As in [3] (q.v.), we can extend the class of modules $A$ to which Theorem 1 applies as follows. If $\Lambda$ is an augmented ring, a $V^{1}$-module for $\Lambda$ is a finite direct sum $A^{\prime}=\sum A_{i}$, where $A_{i}$ is a $\Lambda-k_{i}$-bimodule, finite dimensional over the field $k_{i}$. The $A_{i}$ are called components of $A^{\prime}$. For $j>1$, a $V^{j}$-module is a $\Lambda$-module which is an extension of a $V^{1}$-module by a $V^{j-1}$-module. A $\Lambda$-module is a $W$-module if it is a $V^{j}$-module for some $j$.

Now suppose $\Gamma$ is an augmented ring, $\Lambda$ a subnormal commutative subring, and $A$ a $W$-module for $\Gamma$ such that each component of the $V^{1}$-modules involved in the construction of $A$ satisfies condition (a) or (b) or (c) of Theorem 1. Then if $A^{\Lambda}=0$, $\operatorname{Ext}_{\Gamma}^{r}(Q, A)=0$ for $r \geqslant 0$. The proof of this statement is the same, mutatis mutandis, as that of Theorem 1 of [3]. Indeed, the latter is a consequence of the former since if $\Lambda$ is a supplemented $\mathbf{Z}$-algebra, then condition (c) of Theorem 1 is automatically satisfied because $\Lambda=\mathbf{Z} \oplus I_{\Lambda}[2, \mathrm{X}, \S 1]$.

3. Applications. The reader is referred to [2, IX and XIII] for the cohomology theory of associative and Lie algebras, and to [2, XIV], [5], [6], [7], [8] for the terminology of extension theory used below. (All associative algebras are assumed to have unit element.) We merely note here that if $L$ is a Lie algebra, $\Lambda=L^{e}$ its enveloping algebra, and $A$ an $L$-module, then 


$$
A^{\Lambda}=\{a \in A: l a=0 \text { for all } l \in L\}
$$

since $I_{\Lambda}$ is generated by the image of $L$ in $L^{e}$ [2, p. 268]. Similarly, if $R$ is an associative $k$-algebra, $\Lambda=R \otimes_{k} R^{*}$ its enveloping algebra, and $A$ a two-sided $R$-module, then

$$
A^{\Lambda}=\{a \in A: r a=a r \text { for all } r \in R\}
$$

because $I_{\Lambda}$ is generated by all elements of the form $r \otimes 1-1 \otimes r^{*}, r \in R[2$, IX, Proposition 3.1].

CoRollary 1. Let $L$ be a Lie algebra over a field $k, J$ an abelian subideal of $L$, and $A$ an $L$-module, finite dimensional over $k$, such that

$$
A^{J} \equiv\{a \in A: j a=0 \text { for all } j \in J\}=0 .
$$

Then

$$
H^{r}(L, A)=0 \text { for } r \geqslant 0 .
$$

In particular, any L-kernel with center $A$ is extendible, and this extension is unique (up to equivalence).

Proof. The first conclusion is immediate from Theorem 1 and the remarks preceding that theorem.

The second conclusion follows from the vanishing of $H^{3}(L, A)$ and $H^{2}(L, A)[7]$ (or [8, Lemma 5]) and [8, Theorem 3].

COROLLARY 2. Let $E$ be a Lie algebra over a field, and $A$ a finite dimensional abelian ideal in $E$ such that $E / A$ is abelian. If the center of $E$ is disjoint from $A$, then $E$ splits over $A: E=A \oplus B$ (vector space direct sum), where $B$ is a Lie subalgebra.

Proof. Use Corollary 1 to show that $H^{2}(E / A, A)=0$.

COROLlARY 3. Let $R_{0}$ be a commutative ring, $R$ an associative $R_{0}$-algebra with center $S, k$ a field, and $A$ a two-sided $R$ - $k$-bimodule, finite dimensional over $k$. Suppose at least one of the following conditions holds:

(a) $R$ is a k-algebra (in such a way that (cr)a $=c(r a)$ for all $c \in k, r \in R, a$ $\in A$.

(b) $\left|k_{0}\right|>\operatorname{dim}_{k} A$, where $k_{0}$ is the prime field of $k$.

(c) Every $k$-subspace of $A$ which is invariant under the maps $a \mapsto s a-a s$ for all $s \in S$ is $R$-invariant (as a two-sided module).

If

$$
A^{S} \equiv\{a \in A: \text { sa }=\text { as for all } s \in S\}=0,
$$

then 


$$
H^{r}(R, A)=0 \text { for } r \geqslant 0 .
$$

Proof. Use Proposition 1 (not Theorem 1) with $\Gamma=R \otimes_{R_{0}} R^{*}$. Condition (a) implies condition (a) of Proposition 1 if we make $\Gamma$ a $k$-algebra by defining $c\left(r \otimes r_{1}^{*}\right)=c r \otimes r_{1}^{*}$. Also, it is not hard to see that condition (c) implies condition (c) of Proposition 1.

COROLlary 4. Let $k$ be a field, $R$ a $k$-algebra with center $S, A$ a two-sided $R$ module, finite dimensional over $k$, such that $A^{S}=0$. Then

$$
H^{r}(R, A)=0 \text { for } r \geqslant 0
$$

In particular, if $[f, K]$ is a representation of $R$ with nucleus $A$ (in the sense of Hochschild [5]), then there is a unique (up to equivalence) extension of $K$ by $R$ which gives rise to this representation.

Proof. Use [5, Theorems 5.2 and 6.2] for the second conclusion.

COROLlary 5. Let $E$ be an associative algebra over a field, and $A$ a finite dimensional ideal in $E$ with $A^{2}=0$, such that $E / A$ is commutative. If the center of $E$ is disjoint from $A$, then $E$ splits over $A: E=A \oplus B$ (vector space direct sum), where $B$ is a subalgebra.

Proof. See the proof of Corollary 2 and [2, XIV, Theorem 2.1].

\section{REFERENCES}

1. D. W. Barnes, On the cohomology of soluble Lie algebras, Math. Z. 101 (1967), 343-349. MR 36 \#3836.

2. H. Cartan and S. Eilenberg, Homological algebra, Princeton Univ. Press, Princeton, N.J., 1956. MR 17, 1040.

3. P. M. Curran, Fixed-point-free actions on a class of abelian groups, Proc. Amer. Math. Soc. 57 (1976), 189-193.

4. J. Dixmier, Cohomologie des algèbres de Lie nilpotentes, Acta Sci. Math. (Szeged) 16 (1955), 246-250. MR 17, 645.

5. G. Hochschild, Cohomology and representations of associative algebras, Duke Math. J. 14 (1947), 921-948. MR 9, 267.

6. - Cohomology of restricted Lie algebras, Amer. J. Math. 76 (1954), 555-580. MR 16, 109.

7. _- Lie algebra kernels and cohomology, Amer. J. Math. 76 (1954), 698-716. MR 16, 109.

8. M. Mori, On the three-dimensional cohomology group of Lie algebras, J. Math. Soc. Japan 5 (1953), 171-183. MR 15, 282.

Department of Mathematics, Fordham University, Bronx, New YorK 10458 